\title{
Apoptosis of sensory neurons and satellite cells after sciatic nerve transection in C57BL/6J mice
}

A.L.R. Oliveira
Departamento de Anatomia, Instituto de Biologia,

Universidade Estadual de Campinas, Campinas, SP, Brasil

\section{Correspondence}

A.L.R. Oliveira

Departamento de Anatomia

Instituto de Biologia, Unicamp

Caixa Postal 6109

13083-970 Campinas, SP

Brasil

Fax: + 55-19-3289-3124

E-mail: alroliv@unicamp.br

Publication supported by FAPESP.

Received August 16, 2000

Accepted January 30, 2001

\section{Abstract}

The rate of axonal regeneration, after sciatic nerve lesion in adult $\mathrm{C} 57 \mathrm{BL} / 6 \mathrm{~J}$ mice, is reduced when compared to other isogenic strains. It was observed that such low regeneration was not due just to a delay, since neuronal death was observed. Two general mechanisms of cell death, apoptosis and necrosis, may be involved. By using the terminal deoxynucleotidyl transferase dUTP nick end labeling (TUNEL) technique, we demonstrated that a large number of sensory neurons, as well as satellite cells found in the dorsal root ganglia, were intensely labeled, thus indicating that apoptotic mechanisms were involved in the death process. Although almost no labeled neurons or satellite cells were observed one week after transection, a more than ten-fold increase in TUNEL labeling was detected after two weeks. The results obtained with the C57BL/6J strain were compared with those of the $\mathrm{A} / \mathrm{J}$ strain, which has a much higher peripheral nerve regeneration potential. In $\mathrm{A} / \mathrm{J}$ mice, almost no labeling of sensory neurons or satellite cells was observed after one or two weeks, indicating the absence of neuronal loss. Our data confirm previous observations that approximately $40 \%$ of C57BL/6J sensory neurons die after sciatic nerve transection, and indicate that apoptotic events are involved. Also, our observations reinforce the hypothesis that the low rate of axonal regeneration occurring in $\mathrm{C} 57 \mathrm{BL} / 6 \mathrm{~J}$ mice may be the result of a mismatch in the timing of the neurons need for neurotrophic substances, and production of the latter by non-neuronal cells in the distal stump.

Subsequent to a peripheral lesion, a broad range of events is initiated, both in the proximal and distal stump of the nerve (1). In the distal stump, a process called Wallerian degeneration starts as a local inflammation, being characterized by an extensive recruitment of macrophages which scavenge axon and myelin debris, stimulate Schwann cell proliferation and produce several signaling molecules such as cytokines (2-4). At the
Key words

- TUNEL

- Apoptosis

- Axotomy

- Neuronal death

- Satellite cells

- Isogenic mice same time, the damaged neurons display a series of cytoplasmic alterations characterized as chromatolysis (5). It is known that such a process is the initial morphological step in a cascade of events which will be responsible for neuronal survival as well as axonal regeneration, or for neuronal death, which can occur by apoptosis or necrosis (6). Necrosis is characterized by rapid cell disintegration, cellular swelling, organelle dys- 
function and passive cell disassembly (7). The products of cytoplasmic leakage activate the immune system, leading to macrophage invasion and local inflammation. In contrast, apoptosis is an active process, extensively identified under electron microscopy by a series of morphological alterations (7). It is characterized by early chromatin condensation followed by internucleosomal DNA cleavage, cell shrinkage, reorganization of the cytoskeleton, organelle relocation and production of apoptotic bodies. Apoptosis also requires the transcription of certain genes, protease and endonuclease activation and the expression of phagocytic signals on the cell surface. The entire process ends as a silent and quick cell self destruction, without detectable inflammation (8-10).

It has been reported in the literature that C57BL/6J mice have a lower axonal regeneration potential after a crush lesion when compared to other isogenic strains. Although Lu et al. (11) proposed that such a defect would be a delay rather than a permanent neuronal impairment, Lainetti et al. (12) observed a large percentage of sensitive neuron death four weeks after axotomy. It has been proposed that such neuronal death may be the result of a mismatch in the timing of the neuronal need for trophic substances and their production by the non-neuronal cells in the nerve (13). In this respect, apoptotic mechanisms may be involved, and may be related to the previously described loss of C57BL/6J dorsal root ganglion (DRG) neurons. Taking into account the facts reported above, the aim of the present study was to characterize neuronal loss in C57BL/6J mice using the terminal deoxynucleotidyl transferase dUTP nick end labeling (TUNEL) method for the detection of apoptosis.

Six adult male mice of the C57BL/6J and $\mathrm{A} / \mathrm{J}$ strains were used. The animals were anesthetized $(0.25 \mathrm{~g}$ ketamine and $0.02 \mathrm{~g}$ xylazine dissolved in $5 \mathrm{ml}$ of distilled water, $0.2 \mathrm{ml} / 25 \mathrm{~g}$ body weight, ip) and the left sciatic nerve was exposed and transected at the midthigh level. The proximal stump was ligated with an 8-0 Ethicon stitch and a 2$\mathrm{mm}$ segment of the distal stump was resected in order to avoid regeneration. After one and two weeks $(\mathrm{N}=3$ for each strain and survival time) the animals were sacrificed with an overdose of chloral hydrate $(0.6 \mathrm{mg} / \mathrm{kg}$, ip $)$ and perfused transcardially with $150 \mathrm{ml}$ of $4 \%$ paraformaldehyde in $0.1 \mathrm{M}$ PBS. The L4, L5 and L6 spinal cord segments and L5 DRG were dissected out, left in the same fixative for $24 \mathrm{~h}$, washed in PBS and processed for paraplast embedding. Transverse sections of the spinal cords $(7 \mu \mathrm{m})$ and DRG were obtained, transferred to albumin-coated slides and stored until use. After deparaffinization, endogenous peroxidase was inactivated with $3 \% \mathrm{H}_{2} \mathrm{O}_{2}$ in distilled water for 5 min at room temperature. The slides were transferred to a humidified chamber, the equilibration buffer solution was applied (Oncor, s7110-1) and the slides were incubated for $5 \mathrm{~min}$ at room temperature. The equilibration buffer was shaken off and the TdT enzyme solution (Oncor, s7110-2 and 3) applied for $60 \mathrm{~min}$ at $37^{\circ} \mathrm{C}$. The reaction was stopped by applying the stop/wash solution (Oncor, s7110-4) for $30 \mathrm{~min}$ at $37^{\circ} \mathrm{C}$. The sections were incubated with 2\% BSA (Sigma Chemical Co., St. Louis, MO, USA) for $10 \mathrm{~min}$ and covered for $30 \mathrm{~min}$ with an anti-digoxigenin peroxidase complex (Dako ABC kit, Dako A/S, Glostrup, Denmark). The peroxidase was detected with a 3,3',5,5'diaminobenzidine solution, washed in distilled water, counterstained with hematoxylin and mounted in Entelan (Merck, Darmstadt, Germany). The number of TUNELpositive neurons and satellite cells in the DRG was obtained by counting four alternating sections from each animal. In this way, six fields of each selected section were captured using a video camera (Olympus UCMAD-2) connected to an Olympus BX60 microscope (objective, 100X), and the TUNEL-positive cells counted with the Image Pro Plus 3.0 software (Media Cybernet- 
ics, Baltimore, MD, USA). The total number of neurons and satellite cells of each section was obtained as the sum of the number counted in the six sample areas of each section.

For the spinal motoneurons, alternate sections of the lumbar intumescence were used and processed as described above. The numerical data are reported as mean $\pm \mathrm{SD}$.

One week after sciatic nerve transection, almost no TUNEL labeling was detected in the DRG sections from $\mathrm{A} / \mathrm{J}$ mice. Also, counterstaining revealed no signs of neuronal or satellite cell degeneration. The same pattern was observed two weeks after lesion (Figure 1). With respect to the spinal motoneurons found in the ventral horn of the lumbar spinal cord, no labeling was observed after one or two weeks.

Analysis of sections from C $57 \mathrm{BL} / 6 \mathrm{~J}$ mice obtained one week after transection revealed the absence of neuronal labeling on the contralateral side. Also, a reduced number of TUNEL-positive neurons $(18.7 \pm 6.1)$ and satellite cells $(16.7 \pm 6.1)$ was found in the lesioned side. For the neurons, the number of labeled profiles represented $3 \%$ of the sampled cells. However, two weeks after lesion, a marked increase in TUNEL-labeled sensory neurons $(237.3 \pm 13.6)$ was observed on the ipsilateral side, representing approximately $45 \%$ of the population studied. Such an increase in labeling occurred simultaneously in the satellite cells $(232.7 \pm 41.7$; Figures 1 and 2). No labeling was detected on the contralateral side and the number of normal neurons $(508.0 \pm 21.2)$ was similar to that found one week after lesion (509.1 \pm 10.1).

Although a number of labeled cells showed the basic characteristics of apoptosis, i.e., chromatin condensation, cell shrinkage and fragmentation, some of the TUNELpositive neurons and satellite cells were morphologically normal. No TUNEL labeling was found in the motor nuclei of the lumbar intumescence from $\mathrm{C} 57 \mathrm{BL} / 6 \mathrm{~J}$ mice after one or two weeks.

Peripheral nerve transection in neonatal rats induces an extensive death of lesioned motoneurons in the spinal cord and primary afferent sensory neurons in the DRG $(14,15)$. However, this kind of lesion in adult animals does not result in extensive neuronal death, although a series of alterations known as chromatolysis take place in the cell body (14). In fact, Ekström (16) reported that only a small proportion of the lesioned neurons became apoptotic after sciatic nerve crush in vivo. Also, Schwann cells at the site of injury became TUNEL labeled in the first $2 \mathrm{~h}$ after injury.

The survival rate of adult neurons after disconnection from the target is probably due to the relative independence of certain neurotrophic molecules produced by the nonneuronal cells and target organ. This also means that the neurons from adult individuals have an intrinsic regenerative potential, which is triggered by their disconnection from the target. In this respect, after lesion, adult neurons are able to switch from a transmitting to a growth mode, producing cytoskeleton proteins such as neurofilaments, CGRP and GAP-43 (17). Later, the timing between axonal elongation and the generation of a supportive microenvironment distally to the lesion seems to be essential for neuronal survival and the success of regeneration (18).

Interestingly, C57BL/6J mice have been reported as deficient in regenerating myelinated fibers in the peripheral nerves and DRG (11,12). Although motoneurons are able to regenerate as efficiently as those from other isogenic strains such as A/J, DBA/ $1 \mathrm{~J}$ and $\mathrm{C} 3 \mathrm{H} / \mathrm{HeJ}$, poor sensory neuron survival has been observed. Our results confirm these observations and indicate that apoptotic mechanisms are not present after motoneuron axotomy in $\mathrm{A} / \mathrm{J}$ and $\mathrm{C} 57 \mathrm{BL} / 6 \mathrm{~J}$ mice. In this case, the motoneurons probably receive the necessary amount of trophic substances, such as brain-derived neurotrophic 
factor and ciliary neurotrophic factor to assure cell survival during the critical period without contact with the distal stump and the target organ.

Despite the fact that, in general, sensory neuron death is unlikely after peripheral dam- age in adult animals, it has been proposed as a possible explanation for the observations made in C57BL/6J mice, based on horseradish peroxidase retrograde labeling (12). The present data confirm this hypothesis and show that it is a characteristic of C57BL/6 J

Figure 1 - Transverse sections of lumbar spinal cord and L5 dorsal root ganglia after sciatic nerve transection. A, C57BL/6J mice two weeks after lesion. Observe the number of TUNEL-positive neurons (arrows). Normal neurons are also seen (asterisks). B, Detail of the previous picture showing apoptotic sensory neurons (thick arrows) and satellite cells (thin arrows). C, C57BL/6] normal motoneuron (arrow), two weeks after injury. D, C57BL/6] TUNEL-labeled sensory neuron (arrow) found one week after nerve transection, surrounded by normal cells. E, F, A/J mice one and two weeks after lesion. Observe the absence of TUNEL labeling. Bar $=10 \mu \mathrm{m}$.
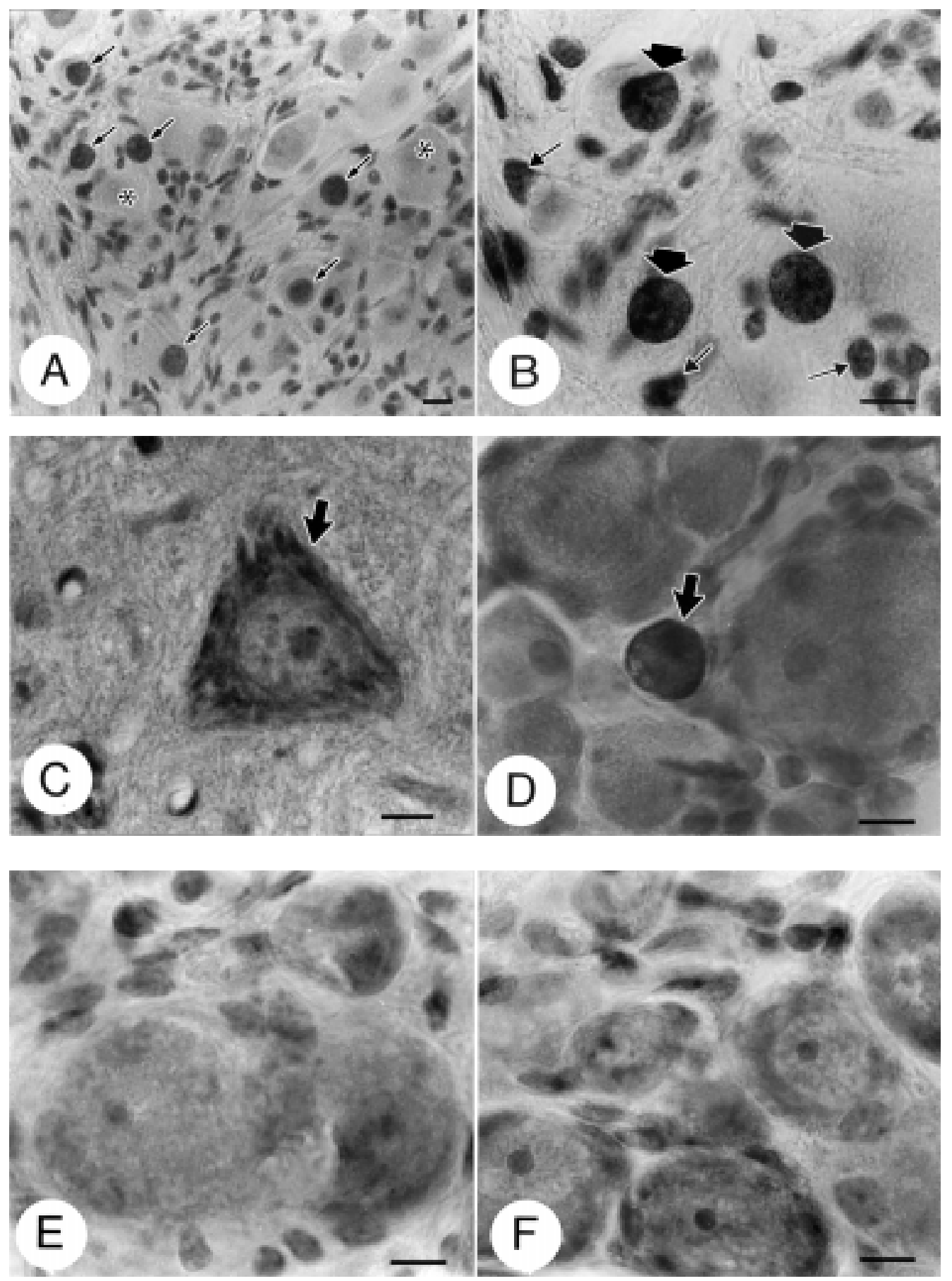
mice, since almost no DRG neuron death was identified after $\mathrm{A} / \mathrm{J}$ sciatic nerve transection, as reported in the literature for NMRI mice (16). Also, our observations indicate that a major proportion of cell death is the result of apoptosis and that satellite cells are also affected. By using the TUNEL technique, it was possible to identify cells with early chromatin fragmentation before any morphological sign of cell death could be noticed. Also, neuronal damage was detected prior to cell death, at least four weeks before the previous report (12), showing that neuronal and satellite cell death occurs around the second week post-lesion. Such timing is consistently different from that reported by Ekström (16) and reinforces the fact that adult C57BL/6J mice have a greater neuronal loss after peripheral lesion compared to other strains.

The low level of TUNEL labeling one week after axotomy may be explained by the fact that, soon after lesion, neurons are able to survive disconnected from the target. Conversely, after this period there is an increase in the need to obtain a certain amount of trophic factors, such as nerve growth factor (NGF), synthesized by non-neuronal cells in the distal stump (19). If such substances are not available, neuronal death can be triggered, also resulting in the loss of satellite cells, as observed two weeks after axotomy. Interestingly, at this point, we found that approximately $45 \%$ of the DRG neurons were TUNEL positive, indicating that a large number of labeled neurons would probably die within a short period of time. This fact can be confirmed by an analysis of the results after horseradish peroxidase tracing, where only about $60 \%$ of the neurons survived (12).

Taken together, our results support the hypothesis that DRG neuron loss is the result

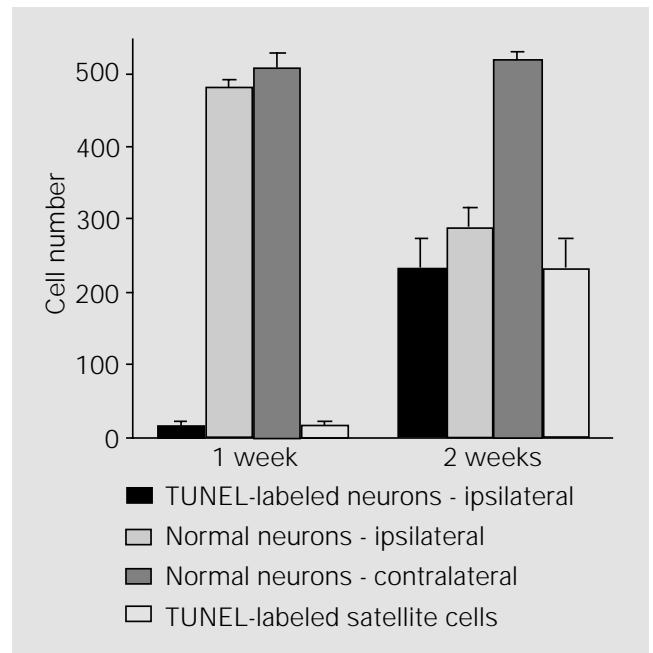

Figure 2 - Number of TUNELlabeled dorsal root ganglion neurons and satellite cells from C57BL/6J mice, one and two weeks after sciatic nerve transection. The number of normal neurons is also presented. Observe the reduced number of TUNEL-positive neurons (3\%) and satellite cells one week after lesion. Two weeks after injury, the number of apoptotic neurons (45\%) and satellite cells was greatly enhanced. of a putative mismatch between the neuronal need for trophic factors and their production by the cells in the distal stump. Also, the possible lack of trophic support results in apoptotic neuronal death, which also includes the satellite cells in the DRG. This hypothesis is in agreement with a previous report which showed that if a predegenerated nerve was grafted to the proximal stump, C57BL/ $6 \mathrm{~J}$ regenerative performance was greatly enhanced (13). Also, if exogenous NGF is administered after sciatic nerve transection, sensory neuron death can be partially reduced (20). Other studies are underway in order to identify possible apoptotic mechanisms involved in C57BL/6J DRG cell loss after peripheral nerve lesion.

\section{Acknowledgments}

The author is grateful to Prof. Glauce A. Pinto and Prof. José Vassallo (Laboratory of Experimental Pathology and Department of Anatomic Pathology, Unicamp) for their support with the TUNEL technique, and also to Cristiano A. Chagas for excellent technical assistance. 


\section{References}

1. Lunn ER, Brown MC \& Perry VH (1990). The pattern of axonal degeneration in the peripheral nervous system varies with different types of injury. J ournal of Neuroscience, 35: 157-165.

2. Bruck W, Bruck C, Marushak B \& Fried RL (1995). Mechanisms of macrophage recruitment in Wallerian degeneration. Acta Neuropathologica, 89: 363-367.

3. Hall SM (1993). Observations on the progress of Wallerian degeneration in transected peripheral nerves of C57BL/Wld mice in the presence of recruited macrophages. J ournal of Neurocytology, 22: 480-490.

4. Taskinen HS, Olsson T, Bucht A, Khademi M, Svelander L \& Royotta M (2000). Peripheral nerve injury induces endoneural expression of IFN-gamma, IL-10 and TNFalpha mRNA. J oumal of Neuroimmunology, 102: 17-25.

5. Peters A, Palay SL \& Webster HF (1991). The Fine Structure of the Nervous System Neurons and their Supporting Cells. 3rd edn. Oxford University Press, New York.

6. Walker NI, Harmon BV, Gobe GC \& Kerr J F (1988). Patterns of cell death. Methods and Achievements in Experimental $\mathrm{Pa}$ thology, 13: 18-54.

7. McConkey DJ , Zhivotovsky B \& Orrenius
S (1996). Apoptosis - molecular mechanisms and biomedical implications. Molecular Aspects of Medicine, 17: 1-110.

8. Adams J M \& Cory Z (1998). The BCL-2 protein family: arbiters of cell sunvival. Science, 281: 1322-1326.

9. Ashkenazi A \& Dixt VM (1998). Death receptors: signaling and modulation. Science, 281: 1305-1308.

10. Thomberry NA \& Lazebnik Y (1998). Caspases: enemies within. Science, 281: 1312-1316.

11. Lu X, Richardson PM, Gervais F \& Skaeme $E$ (1990). A deficiency of axonal regeneration in C57BL/6] mice. Brain Research, 510: 144-146.

12. Lainetti RD, Pereira FC \& Da-Silva CF (1995). Reduced sensory neuron regeneration by C57BL/6J mice. Brazilian J ournal of Medical and Biological Research, 28: 781-785.

13. Oliveira ALR \& Langone F (2000). Nonneuronal cells are not the limiting factor for the low axonal regeneration in C57BL/ 6] mice. Brazilian J ournal of Medical and Biological Research, 33: 1467-1475.

14. Lowrie MB \& Vbrová G (1992). Dependence of postnatal motoneurons on their targets: review and hypothesis. Trends in Neurosciences, 15: 80-84.

15. Whiteside G, Doyle CA, Hunt SP \&
Munglani R (1998). Differential time course of neuronal and glial apoptosis in neonatal rat dorsal root ganglia after sciatic nerve axotomy. European J ournal of Neuroscience, 10: 3400-3408.

16. Ekström AR (1995). Neurones and glial cells of the mouse sciatic nerve undergo apoptosis after injury in vivo and in vitro. NeuroReport, 6: 1029-1032.

17. Fu SY \& Gordon T (1997). The cellular and molecular basis of peripheral nerve regeneration. Molecular Neurobiology, 14: 67-116.

18. Taira E, Takaha N \& Miki N (1993). Extracellular matrix proteins with neurite promoting activity and their receptors. Neuroscience Research, 17: 1-8.

19. Funakoshi H, Frisén J, Barabany G, Timmusk T, Zachrisson O, Verge VMK \& Persson H (1993). Expression of mRNAs for neurotrophins and their receptors after axotomy of the sciatic nerve. J ournal of Cell Biology, 123: 455-464.

20. Da-Silva CF \& Langone F (1989). Addition of nerve growth factor to the interior of a tubular prosthesis increases sensory neuron regeneration in vivo. Brazilian J ournal of Medical and Biological Research, 22: 691-694. 